\title{
Differences between literates and illiterates on symbolic but not nonsymbolic numerical magnitude processing
}

\author{
Samar Zebian • Daniel Ansari
}

Published online: 27 October 2011

(C) Psychonomic Society, Inc. 2011

\begin{abstract}
The study of numerical magnitude processing provides a unique opportunity to examine interactions between phylogenetically ancient systems of semantic representations and those that are the product of enculturation. While nonsymbolic representations of numerical magnitude are processed similarly by humans and nonhuman animals, symbolic representations of numerical magnitude (e.g., Hindu-Arabic numerals) are culturally invented symbols that are uniquely human. Here, we report a comparison of symbolic and nonsymbolic numerical magnitude processing in two groups of participants who differ substantially in their level of literacy. In this study, level of literacy is used as an index of level of school-based numeracy skill. The data from these groups demonstrate that while the processing of nonsymbolic numerical magnitude (numerical distance effect) is unaffected by an individual's level of literacy, the processing of Hindu-Arabic numerals differs between literate and illiterate individuals who live in a literature culture and have limited symbolic recognition skills. These findings reveal that nonsymbolic numerical magnitude processing is unaffected by enculturation, while the processing of numerical symbols is modulated by literacy.
\end{abstract}

Keywords Literacy · Numerical cognition · Nonsymbolic . Symbolic $\cdot$ Numerical magnitude $\cdot$ Enculturation

\author{
S. Zebian $(\square)$ \\ Social Science Department, Lebanese American University, \\ Beirut, Lebanon \\ e-mail: samar.zebian@lau.edu.lb \\ D. Ansari $(\triangle)$ \\ Numerical Cognition Laboratory, \\ Department of Psychology \& Graduate Program in Neuroscience, \\ University of Western Ontario, \\ London, Canada \\ e-mail: daniel.ansari@uwo.ca
}

\section{Introduction}

Numerical magnitude processing is thought to be grounded in an ancient evolutionary system for the representation and processing of numerical magnitude in nonsymbolic format (sets of items) that is subserved by similar brain structures in both humans and nonhuman primates (e.g., Nieder \& Dehaene, 2009).

Over the course of cultural history, humans have invented symbolic systems for the representation of numerical magnitude. It is commonly assumed that numerical symbols are mapped onto preexisting, phylogenetically ancient, nonsymbolic numerical magnitude representations (e.g., Verguts \& Fias, 2004); however, the nature of the relationship between these representations is not well understood (e.g., Ansari, 2008).

In the present study, we explore the relationship between symbolic and nonsymbolic processing by comparing the performance of illiterate and the participants on measures of symbolic and nonsymbolic numerical magnitude comparison. In the present study, literacy skill was used as an index of level of education, or more precisely, it is a correlate of one's level of school-based symbolic numeracy skill. Literacy has been used as a proxy for education in many other studies ( $\mathrm{Li}$ et al., 2006; Petersson, Reis, \& Ingvar, 2001). Although it would be ideal to directly assess numeracy skills, there are no known normed numeracy assessment tools for illiterate participants (see Ardila et al., 2010).

Thus, the present study represents a controlled experimental study with carefully matched groups, which examines the presently unresolved question of whether an individual's learning trajectory-specifically, their literacy skill-differentially modulates nonsymbolic and symbolic representations of numerical magnitude. By doing so, this investigation can constrain current theoretical understand- 
ing of the relationship between symbolic and nonsymbolic numerical magnitude processing and can directly probe the role played by processes of enculturation.

Evidence for the cultural invariance of nonsymbolic numerical magnitude representation has been suggested through offline studies of illiterates who are speakers of languages that do not have count words for numbers greater than 3-4 (Butterworth, Reeve, Reynolds, \& Lloyd, 2008; Gordon, 2004). Despite a rudimentary counting system, these individuals exhibit performance on offline nonsymbolic magnitude processing tasks (spatial matching tasks, memory for counters, cross-modal matching, and nonverbal approximate addition) that is very similar to that of speakers whose language has a symbolic representation of number.

There has been one documented attempt to administer a conventional speeded number comparison task with large numerosities (20-80) to Munduruku illiterates and bilinguals who spoke Portuguese and had some basic education (Pica, Lemer, Izard, \& Dehaene, 2004). Attesting to the difficulty of reaction time research with illiterates, Pica et al. reported that some of the participants refused to use the computer, while others could not do the task in a way that yielded valid data. As a result, accuracy data, rather than reaction time data, were the main assessment of nonsymbolic magnitude processing. Although studies using accuracy as the dependent variable provide a legitimate assessment of nonsymbolic processing, the concurrent assessment of both accuracy and reaction time data affords a systematic evaluation of strategic processing, such as potential group differences in speed-accuracy trade-offs. Hence, the present comparison of symbolic and nonsymbolic number processing between literate and illiterate participants recorded both accuracy and reaction time data.

Perhaps more important, the present investigation also differs from the available evidence on illiterate numerical cognition in three other ways. First, we examined illiterates who live in mainstream literate society and who are consequently exposed to symbolic representations of numerical magnitude and have some rudimentary symbolic recognition and conceptualization skills, in contrast to previously studied individuals, who lived in remote illiterate communities. Therefore, the present samples afforded a unique examination of both symbolic and nonsymbolic magnitude processing; previous studies were able to assess the effects of literacy only on nonsymbolic magnitude processing, since the illiterates tested were not exposed to symbolic representations of numerical magnitude. Thus, to the best of our knowledge, this is the first investigation of the effects of literacy/bilinguals on both symbolic and nonsymbolic numerical magnitude processing. Furthermore, although our illiterates were able to recognize numerals, they did not have the same level of education as their literate peers and, thus, had less formal experience with numerical symbols and the processing of their semantic referents. The third novelty of this study is that it controlled for differences in cultural environment, occupation, and so forth, which were left to covary with literacy in previous studies.

It could be posited that a less laborious approach to exploring the effects of literacy on symbolic and nonsymbolic numerical magnitude processing is to assess agerelated changes in these competencies through crosssectional studies of groups of differently aged children. Such studies have revealed that the slope relating reaction times and numerical distance for both symbolic and nonsymbolic comparison tasks decreases over developmental time, suggesting a decrease in the overlap of numerical magnitude representations (Holloway \& Ansari, 2009; Sekuler \& Mierkiewicz, 1977). Such studies indicate that both symbolic and nonsymbolic numerical magnitude processing are affected by learning and development. What these studies, however, cannot elucidate is whether such changes are the product of maturation (e.g., changes in brain functions supporting magnitude comparison) or experience (e.g., the acquisition of literacy and other educational processes).

In view of the above, the present study will help constrain theories concerning the effects of literacy and education on the processing of symbolic and nonsymbolic representations of numerical magnitude and their relationship. More specifically, it might be hypothesized that the acquisition of literacy skills and consequent greater formal operations with numerical symbols would lead to changes in nonsymbolic numerical magnitude processing. If this were true, literates and illiterates should differ on both symbolic and nonsymbolic numerical magnitude processing. If, however, the effects of literacy are specific for symbolic numerical magnitude processing, no group differences for nonsymbolic processing should be found.

\section{Method}

\section{Participants}

The minimally literate (ML) group consisted of 11 male participants who could not read Arabic words but could read single-digit Hindi-Arabic numerals and, as such, had at least rudimentary symbolic numeral recognition skills. Pilot testing indicated that all the ML participants included in the final sample could also keep track of small quantities, count, and engage in simple arithmetic using regrouping methods, and counting up strategies on their fingers. Despite their symbolic recognition skills and 
their basic arithmetical skills, it is unlikely that their symbolic magnitude representations were similar to that of literates, given the large differences in formal educational experience between the groups.

The ML group reported having had no more than 1 year of schooling. The highly literate (HL) group of 11 males reported having attended school for more than 10 years. The HL group was administered an Arabic language test, which all of the members passed. The illiterate sample consisted of individuals who could not do the Arabic language test and only passed level 1 of the test of Arabic numeral knowledge and literacy described below.

The socioeconomic background of the two groups was comparable, given that all participants were originally from the agrarian parts of Northern Syria and were working in Lebanon on a seasonal basis. Among the ML group, 5 participants were laborers, 3 were painters, and 3 worked in construction. Among the HL group, 7 participants were laborers, 2 were painters, and 2 worked in construction. The participants were recruited with the help of a Syrian-Lebanese research assistant who was trusted among the migrant worker population.

The final group of 22 participants describe above was drawn from a larger group of 65 participants. Participants were excluded from the final research sample and all analyses if they met at least one of the following criteria: (1) those who still felt uncomfortable participating in the study after they had been familiarized with the computer and after they had completed three rounds of practice trials, (2) participants who reported being too tired to continue the study, (3) university students, (4) participants with inconsistent performance on the literacy assessment (described below), and (5) functionally illiterate participants who obtained a literacy level higher than level 2, as described below.

These exclusion criteria safeguarded the validity of the data by ensuring that all participants could perform the tasks, and they allowed us to differentiate between individuals with varying levels of functional illiteracy, as well as limiting the sample to individuals with a similar socioeconomic background. The final sample of 22 participants should be considered a theoretical sample and not a representative sample.

\section{Literacy assessment}

Participants' literacy skills were tested in a different way depending on whether they claimed to be literate or illiterate. Literate participants were given a two-part Arabic language test, which assessed reading comprehension, writing skills, and grammatical knowledge. The first part of the test assessed reading comprehension and writing skills and required participants to read a 300-word passage, summarize it, and provide a written critical reflection. The second part required participants to place diacritics on every letter in an unpointed paragraph. Correct placement of diacritics is an objective means of assessing phonological, semantic, and grammatical knowledge in Arabic. Participants were included in the literate group if they were able to complete both parts of this test.

Participants who claimed to be illiterate were offered the language test described above to verify that they were indeed illiterate. If they could not do the language test, their symbolic Hindu-Arabic numeral recognition and their level of literacy skill were assessed as follows:

1. Naming of visually presented Arabic numerals 1-9. All nine Arabic numerals had to be named correctly before the next level was assessed.

2. Participants were asked to name 15 randomly selected letters from the Arabic alphabet. All 15 letters had to be correctly identified before the next level was assessed.

3. For this level, participants were required to read pairs of orthographically and phonetically similar Arabic letters. The correct identification of these letters is a significant milestone for Arabic learners. There are nine

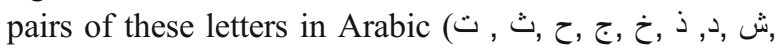

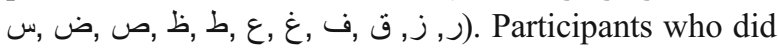
not err in this assessment continued the test.

4. At this level, we assessed the number of high-frequency words that could be read. Ten standard high-frequency words were selected- e.g., in, Allah, from, to, man. All 10 words had to be identified before going on to the next assessment.

5. We assessed the number of low-frequency words that could be read- e.g., policeman, argument, replied. If all 10 low-frequency words were correctly named, the last assessment was administered.

6. In order to assess online comprehension, the participants were required to read one sentence without stopping and then state its meaning without referring back to the text. If they could do this accurately and without stopping to reread the text, they were considered to have achieved level 6.

Only participants who reached level 1 but could not complete items for any of the other levels were included in the group of illiterates.

Stimuli and procedure

Participants were tested in either a laboratory setting or a quiet room in the participants' home, using a 12-in. laptop computer. Participants' torsos were $20-30 \mathrm{~cm}$ from the screen. The stimuli were presented using SuperLab Pro software. Participants pressed one of two keys ("m" or " $\mathrm{z}$ "), depending on which side of the screen displayed the larger of two single-digit Hindu-Arabic numerals (symbolic 
Table 1 Stimuli by distance used for both the symbolic and nonsymbolic magnitude comparison tasks

\begin{tabular}{lll}
\hline $\begin{array}{l}\text { Numerical Distance } \\
\text { Between Numeral Pairs }\end{array}$ & $\begin{array}{l}\text { Left-right Numeral Pairs } \\
\text { With Small Numeral on the Left }\end{array}$ & $\begin{array}{l}\text { Right-Left Numeral Pairs With } \\
\text { Small Numerals on the Right }\end{array}$ \\
\hline 1 & 2,$3 ; 3,4 ; 4,5 ; 5,6 ; 7,8 ; 8,9$ & 3,$2 ; 4,3 ; 5,4 ; 6,5 ; 8,7 ; 9,8 ;$ \\
2 & 2,$4 ; 3,5 ; 4,6 ; 5,7 ; 6,8 ; 7,9$ & 4,$2 ; 5,3 ; 6,4 ; 7,5 ; 8,6 ; 9,7$ \\
3 & $2,5: 3,6 ; 4,7 ; 4,7 ; 5,8 ; 6,9$ & 5,$2 ; 6,3 ; 7,4 ; 7,4 ; 8,5 ; 9,6$ \\
4 & 1,$5 ; 2,6 ; 2,6 ; 3,7 ; 4,8 ; 5,9$ & 5,$1 ; 6,2 ; 6,2 ; 7,3 ; 8,4 ; 9,5$ \\
5 & 1,$6 ; 2,7 ; 2,7 ; 3,8 ; 3,8 ; 4,9$ & 6,$1 ; 7,2 ; 7,2 ; 8,3 ; 8,3 ; 9,4$ \\
6 & 1,$7 ; 1,7 ; 2,8 ; 2,8 ; 3,9 ; 3,9$ & 7,$1 ; 7,1 ; 8,2 ; 8,2 ; 9,3 ; 9,3$ \\
7 & 1,$8 ; 1,8 ; 1,8 ; 2,9 ; 2,9 ; 2,9$ & 8,$1 ; 8,1 ; 8,1 ; 9,2 ; 9,2 ; 9,2$ \\
Total & 84 numeral pairs & 84 numeral pairs \\
\hline
\end{tabular}

magnitude condition) or the more numerous of two arrays of squares (nonsymbolic magnitudes). Each numeral occupied a space of $8 \mathrm{~mm}$ (height) $\times 6 \mathrm{~mm}$ (width) and was presented in Times New Roman font. Participants were instructed to respond as quickly and as accurately as possible. Stimuli remained on the screen for up to 5,000 $\mathrm{ms}$ or until a response was given. The long stimulus presentation ensured that both our ML and HL participants, all of whom reported having no computer experience, had sufficient time to process the numerals and to coordinate their responses with the onset of the stimulus events in the allocated time period. ${ }^{1}$ Between trials, a central fixation dot appeared for 1,000 ms. In both the symbolic and nonsymbolic tasks, participants were presented with a total of 84 trials consisting of combinations of one to nine squares or the Hindu-Arabic numerals 1 to 9 . Forty-two trials consisted of left-right ordered stimuli (i.e., 18 ), while the remaining 42 consisted of right-left trials $(81)$. For each block of symbolic numerals and nonsymbolic arrays, 15 practice trials were performed. The numerical distance between stimuli ranged from 1 to 7 (numerical distances of 8 were not used), with 12 comparison trials per distance (see Table 1). In the symbolic task, the numeral pairs were displayed in the middle of the screen and were $15.5 \mathrm{~cm}$ apart.

In the nonsymbolic condition, the individual area, total area, and density of the squares were systematically varied to ensure that nonnumerical cues could not be used to make a correct decision. Specifically, in 4 of each 12 distance pairings, the smaller numerosity was presented with the larger overall area. In another four exemplars of a particular distance, the smaller numerosity had a smaller overall area. The final 4 pairings presented both the small and large numerosities with equal overall areas. Within each group of four pairs, two of the small numerosity stimuli had larger density, and two had smaller density, as compared with the large numerosity with which each was paired. In addition, individual square sizes were varied over all stimuli. The

\footnotetext{
${ }^{1}$ During the piloting of the tasks, a 3,000-ms stimulus presentation was used, but it resulted in a high percentage of nonresponses.
}

symbolic and nonsymbolic stimuli used in this study are identical to those that have been used in several behavioral studies of symbolic and nonsymbolic number processing (e.g. Holloway \& Ansari, 2009).

\section{Results}

On a participant-by-participant basis, reaction times for erroneous trials were removed, and trials with reactions times greater or less than 2 standard deviations were removed. In addition, any trials where the reaction times exceeded 4,000 ms were also excluded from further analysis. In the ML group, $4.2 \%$ of trials in the symbolic and $5.5 \%$ of the trials in the nonsymbolic task were excluded, on average. The mean percentage of outliers in the HL group was $4.8 \%$ of symbolic and $4.4 \%$ of nonsymbolic trials.

\section{Accuracy}

A task (symbolic vs. nonsymbolic) $\times$ group (illiterates vs. literates) repeated measures ANOVA on the number of correct responses revealed a main effect of task, $F(1,20)=58.8, p<$ .001 , but no significant interaction between the factors, $F(1$, $20)=3.0, p=.55$, and no main effect of group, $F(1,20)=$ 4.104, $p=.056$. Independent-samples $t$-tests indicated that, across the two groups, more errors were made during nonsymbolic than during symbolic number comparison, $t$ $(21)=7.8, p<.001$. Furthermore, the two groups did not differ in accuracy for the symbolic condition, $t(20)=-0.155$, $p=.14$, or the nonsymbolic comparison, $t(20)=-2.1, p=$ .062. Since accuracy was both high and not significantly different between groups, the subsequent analyses were conducted for reaction times only. To assess the presence of any speed-accuracy trade-offs, we calculated the correlations between mean accuracy and mean reaction times for symbolic and nonsymbolic conditions separately for the groups of literates and illiterates. None of these correlations were found to be significant (all $p \mathrm{~s}>.05$ ). 
Table 2 Descriptive data for participants in the minimal- and high-literacy groups. Values in brackets represent the standard deviations

\begin{tabular}{lllllll}
\hline & $\begin{array}{l}\text { Chronological } \\
\text { Age (Years) }\end{array}$ & $\begin{array}{l}\text { Years in } \\
\text { School }\end{array}$ & $\begin{array}{l}\text { Mean Reaction Time } \\
\text { Intercept Symbolic }\end{array}$ & $\begin{array}{l}\text { Mean Reaction Time } \\
\text { Intercept Nonsymbolic }\end{array}$ & $\begin{array}{l}\% \text { Correct } \\
\text { Symbolic }\end{array}$ & $\begin{array}{l}\% \text { Correct } \\
\text { Nonsymbolic }\end{array}$ \\
\hline $\begin{array}{l}\text { Minimal } \\
\text { literacy (ML) }\end{array}$ & $33(11)$ & $0.5(0.5)$ & $1,484(296.5)$ & $1,395(343.0)$ & $92(5.8)$ & $85(5.4)$ \\
\begin{tabular}{l} 
High literacy (HL) \\
\hline
\end{tabular} & $29(5.5)$ & $12(1.2)$ & $1,002(128.0)$ & $1,009(274.6)$ & $95(1.0)$ & $89(3.0)$ \\
\hline
\end{tabular}

Mean reaction time intercepts

A repeated measures ANOVA was run to assess the effects of format (symbolic vs. nonsymbolic) and group (HL vs. ML) on the reaction time intercepts (see Table 2 for means). The effect of format was found to be nonsignificant, $F(1,20)=$ $0.53, p=.407$, as was the interaction between format and group, $F(1,20)=0.72, p=.40$. The main effect of group was found to be significant, $F(1,20)=18.4, p<.001$. Independent-samples $t$-tests confirmed that illiterates were slower in both the symbolic, $t(20)=4.9, p<.001$, and the nonsymbolic, $t(20)=3.7, p<.001$, comparison tasks.

\section{Distance effects}

In order to enumerate the relationship between distance and reaction time, a linear regression was calculated for each participant, with distance (1-7) as the independent factor and reaction time as the dependent factor. From this calculation, the unstandardized beta coefficient was extracted and used to represent the slope relating reaction time and numerical distance for each individual. This procedure was undertaken separately for the symbolic and nonsymbolic conditions for each participant. Since the numerical distance effect is characterized by an inverse relationship between reaction times and numerical distance (see Fig. 2), the slopes for this effect are expected to be negative. One-sample $t$-tests confirmed that the slope relating numerical distance and reaction times was significantly different from zero and negative (see Fig. 1) for

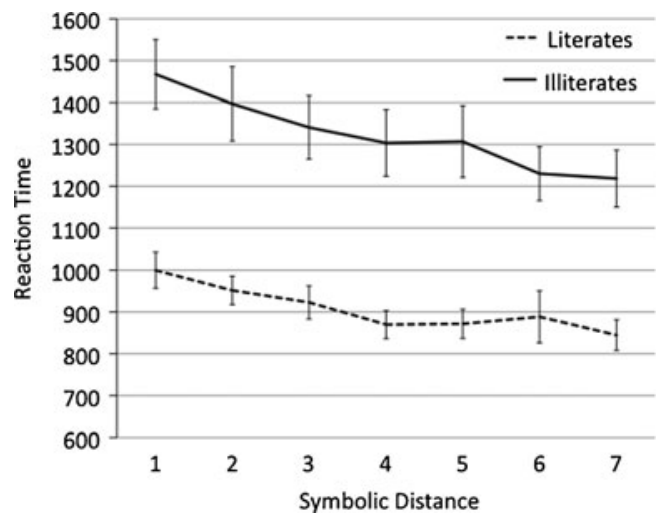

Fig. 1 Mean reaction times for symbolic (Arabic numerals) distances 1-7 for the literate and illiterate groups of participants. Error bars represent the standard errors of the means both symbolic and nonsymbolic in the illiterate and literate groups (all $p \mathrm{~s}<.01$ ). These findings, therefore, confirm a significant numerical distance effect in both groups for each task.

Independent-samples $t$-tests were calculated to compare the slopes relating symbolic and nonsymbolic distance effects between groups. The results of these indicated that the ML group had a significantly steeper symbolic slope, as compared with the HL group, $t(20)=-2.4, p=.03$. The HL and ML groups did not show a significant difference in their nonsymbolic slope, $t(20)=-0.25, p=.80$ (see Fig. 3). ${ }^{2}$

Given the relatively small number of participants, we calculated Bayes factors for both two-sample $t$-tests, using the procedure outlined by Rouder, Speckman, Sun, Morey, and Iverson (2009). For the between-group analysis of the slope of the symbolic distance effect, the analysis revealed a Bayes factor of -.39, representing evidence for a model other than the null hypothesis (alternative model). In contrast, the Bayes factor for the between-group analysis of the slope of the nonsymbolic distance effect was found to be 3.25 , which represents evidence for the null hypothesis. Thus, the calculation of Bayes factors supports the presence of a group difference for the symbolic, but not the nonsymbolic, slope of the distance effect on reaction times.

An inspection of the data in Figs. 1 and 2 suggests that while the relationship between numerical distance and reaction times is linear for the illiterates, it appears to be curvilinear for the group of literates. Consequently, we ran a within-subjects contrast to investigate the significance of

\footnotetext{
$\overline{2}$ There may be some concern that the removal of trials on which the reaction times were 2 standard deviations below or above a participants' mean reaction time might have affected the results. In order to address this concern, we evaluated group differences in the slope relating distance and reaction times, using the unedited data (no cutoff). This analysis replicated the existing findings of a significant difference between illiterate and literate participants for the symbolic $(p=.017)$, but not the nonsymbolic, distance effect $(p=.117)$. However, when running the independent samples $t$-tests to compare symbolic and nonsymbolic distance effects between groups, we noticed that Levene's tests of equality of variances were significant. In view of this, we ran this analysis using the nonparametric, independent-samples Mann-Whitney $U$ test. This analysis also revealed a significant difference between groups for the symbolic $(p=.033)$, but not the nonsymbolic $(p=.178)$, distance effect. Therefore, even when no cutoff is applied, the raw data support our central conclusions. Thus the cutoff does not have an impact beyond making the data suitable for the use of parametric statistical testing.
} 


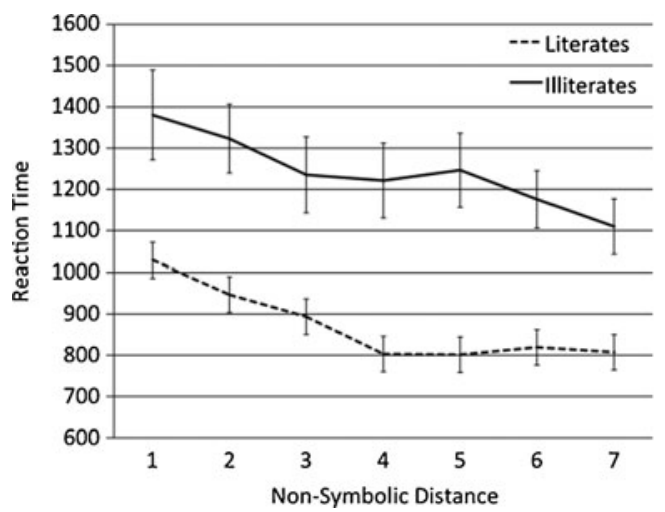

Fig. 2 Mean reaction times for nonsymbolic (arrays of squares) distances 1-7 for the illiterate and illiterate groups of participants. Error bars represent the standard errors of the means

linear and nonlinear effects of distance (seven levels). For the symbolic distance effect, these analyses revealed that, in the illiterate participants, both linear, $F(1,10)=20.6, p=$ .001 , and quadratic, $F(1,10)=15.7, p=.003$, explained significant variance in the data. In contrast, for the illiterates, only the linear contrast was found to be significant, $F(1,10)=56.3, p=.001$. The same was found to be true for the nonsymbolic distance effect, where both linear, $F(1,10)=18.2, p=.002$, and quadratic, $F(1,10)=$ $22.4, p=.001$, contrasts were significant for the literates, while only the linear contrast was found to be significant for the illiterates, $F(1,10)=24.2, p=.001$. While these data show that the relationship between numerical distance and reaction time is significantly curvilinear in the literates but solely linear in the illiterate group, these differences are unlikely to explain the group differences in the degree of the symbolic, but not nonsymbolic, distance effects. This is due to the following reason: The group difference in the extent to which the distance effect was curvilinear was found for both the symbolic and nonsymbolic comparison tasks, while the group difference in the strength of the distance effect was significant only for the symbolic task. If the difference in the linearity of the relationship were the reason behind the observed group effect in the distance effect, such a difference should have been observed for both the symbolic and nonsymbolic comparisons, rather than for the symbolic only.

In order to address this issue statistically, we ran an additional set of analyses to probe the group differences in the symbolic and nonsymbolic distance effect. Specifically, two repeated measures ANOVAs were run to evaluate the distance effect for nonsymbolic and symbolic comparison and their interaction with group. To do this, the average reaction times for distances 1 and 2 were calculated to represent the RTs for the smallest distances, and the mean RTs for distances 6 and 7 were calculated to reflect the largest distances. Consistent with the symbolic slope

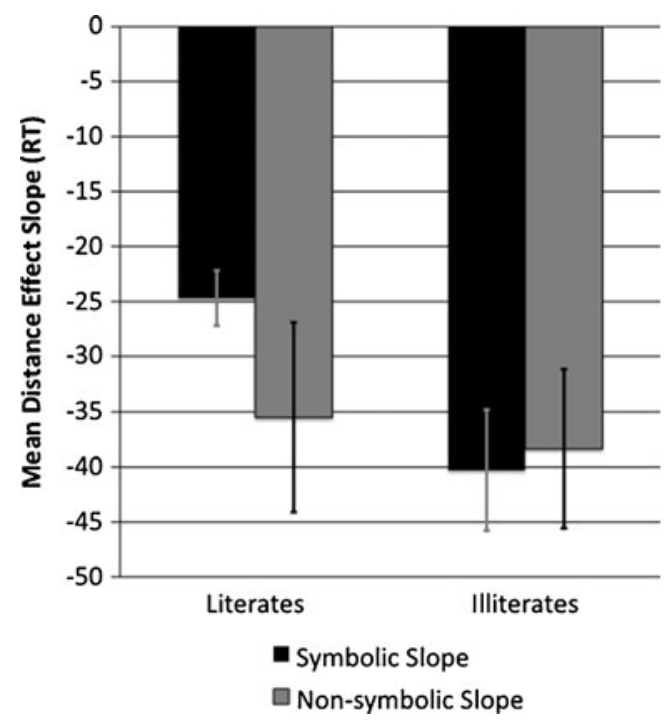

Fig. 3 Bar charts for the mean slope for the relationship between reaction time and symbolic and nonsymbolic numerical distance for the group with minimal literacy and their high literacy peers. Error bars represent the standard errors of the means

analyses reported above, a main effect of distance (small vs. large) was obtained, $F(1,20)=58.78, p<.001$. Critically, the interaction between distance and group was also significant for the symbolic task, $F(1,20)=5.7, p=.027 .^{3}$ The analysis of the nonsymbolic condition yielded a significant main effect of distance, $F(1,20)=44.48, p<.001$, but no significant interaction between distance and group, $F(1,20)=0.35, p=$

\footnotetext{
$\overline{3}$ Table 1 shows that the number of repeated trial pairs increases with numerical distance. Therefore, it is possible that stimulus repetition affected illiterates and literates differentially and, thereby, influenced the distance $\times$ group interaction observed for the symbolic condition (in which, unlike in the nonsymbolic condition, exact stimuli were repeated). To address this, we included repetition as a factor in a follow-up analysis of the ANOVA above, in which distance was held constant while repetition was varied. Specifically, while distances 1 versus 6 and 2 versus 7 both differ by a numerical distance of 5 , the number of repeated trials differs beween them. For 1 versus 6 , there are three more unique, nonrepeated trials for distance 6 , as compared with distance 1 . In contrast, for 2 versus 7, there are four more unique trials for distance 7 , as compared with distance 2 . Thus, it is possible to model for the main effects and interactions between distance and repetition by establishing whether the distance effect differs for 1 versus 6 , as compared with 2 versus 7, given the differential amounts of repetition. Consistent with the ANOVA presented above, a main effect of distance was found, $F(1,20)=58.7, p<.001$. Furthermore, the main effect of repetition was also significant, $F(1,20)=7.7, p=.012$. Importantly, while a significant interaction between numerical distance and group was obtained, $F(1,20)=5.7, p=.027$, no interaction between repetition and group $F(1,20)=0.025, p=.87$, and repetition and distance, $F(1$, $20)=1.0, p=.32$, and no three-way interaction between repetition, distance, and group, $F(1,20)=0.03, p=.39$, were obtained. This analysis therefore shows that when distance is held constant and repetition is varied, repetition does not interact with group, nor does it lead to a diminished group $\times$ distance interaction. Thus, while distance and repetition were correlated in the present design, this correlation cannot explain the differential strength of the symbolic distance effect between literate and illiterate participants.
} 
.55. These results are, therefore, consistent with those of the linear slope analysis and confirm that the numerical distance effect differs between illiterate and literate participants for the symbolic, but not for the nonsymbolic, comparison condition, even when one does not compare the linear slope directly.

\section{Discussion}

The study of numerical magnitude processing and representation has demonstrated the existence of a system for nonsymbolic numerical magnitude processing (see Nieder \& Dehaene, 2009). Furthermore, the performance pattern of illiterate individuals on offline nonsymbolic magnitude tasks is virtually indistinguishable from that of individuals from literate cultures where symbolic representation of number and counting sequences are explicitly taught (Butterworth et al., 2008; Pica et al., 2004). This suggests that the nonsymbolic representation of numerical magnitude may not be subject to enculturation. In literate cultures, however, humans have developed symbolic systems for the representation of numerical magnitude, such as Hindu-Arabic numerals.

In the present study, a direct assessment of the effect of literacy/education on the processing of symbolic and nonsymbolic numerical magnitude was made by contrasting two groups of adult participants who came from similar cultural and socioeconomic backgrounds but differed dramatically in how literate they were. Our data revealed that individuals with minimal levels of literacy and rudimentary symbolic numeral processing skills exhibited a steeper effect of numerical distance in a symbolic number comparison task. In contrast, for the numerical magnitude comparison task with nonsymbolic stimuli, no difference between the two groups could be found. These findings, therefore, suggest that symbolic and nonsymbolic numerical magnitude processing is differentially affected by literacy. In addition, these results do not support the hypothesis that literacy and education lead to changes in nonsymbolic numerical magnitude processing. By doing so, the present data also further constrain the interpretation of available developmental evidence. Developmental studies suggest that both symbolic and nonsymbolic numerical magnitude processing change over the course of learning and development. The present findings raise the possibility that developmental differences in symbolic numerical magnitude processing are mediated by literacy and education, while ontogenetic changes in nonsymbolic numerical magnitude processing are largely the product of maturation, rather than enculturation.

It is important to note that the group difference in the symbolic distance effect is characterized by a subtle difference in the magnitude of the distance effect on reaction times, rather than a categorical difference where one group exhibits a distance effect, while the other one does not. This differences in the strength of the distance effect is important in light of the developmental literature showing that, in comparison with young children, older children exhibit a smaller effect of numerical distance (Sekuler \& Mierkiewicz, 1977) and that relatively smaller distance effects are associated with higher scores on tests of mathematical competence (Holloway \& Ansari, 2009).

It is important to note that participants in both groups demonstrated equal ability in correctly identifying HinduArabic numerals, as measured by level 1 in the literacy assessment for the illiterate participants (see the Method section above). Thus, the difference in the effect of the symbolic numerical distance effect between the groups cannot be explained by numeral decoding processes but is more likely a result of processes related to the fluent, semantic processing of Arabic numerals that results from becoming literate. Moreover, the effects observed cannot be explained by between-group differences in accuracy or reaction time, since the groups did not differ significantly in accuracy for either symbolic or nonsymbolic tasks (although a nonsignificant trend was observed for the nonsymbolic data). Additionally, the illiterate participants, in comparison with their literate peers, were significantly slower for both tasks, and this was likely a result of their unfamiliarity with speeded computerized tasks, and not magnitude-related processes.

With these observations as a backdrop, the present data cannot fully exclude the possibility that the strategies used for enumeration and comparisons of the nonsymbolic arrays may be different for literates and illiterates. This is the case for all previous studies comparing nonsymbolic numerical magnitude processing between illiterates and literates. While the absence of a difference in the slope relating reaction time for nonsymbolic numerical distance speaks against this possibility, future research may benefit from comparing literates and illiterates on speeded nonnumerical tasks to verify that magnitude processing, and not other types of processes, explain the difference between symbolic and nonsymbolic processing in illiterates. It would also be helpful to ascertain whether there may be subtle differences in the strategies employed for magnitude comparison by these groups.

Current theoretical accounts of the numerical distance effect suggest that it arises from the comparison stage, rather than reflecting numerical magnitude representations themselves (Van Opstal, Gevers, De Moor, \& Verguts, 2008). In view of this, we speculate that the group differences in symbolic number representation reflect differences in the representation-to-output connections related to the symbolic magnitude comparison process. We contend that increased experience with Arabic numerals and symbolic numeracy practices (mediated by the process of becoming literate) strengthens these connections, partic- 
ularly for the small distances, thereby reducing the size of the distance effect. However, the present study cannot exclude the possibility that the group differences reflect differences in the overlap of symbolic numerical magnitude representation, rather than changes in the representation-tooutput connections. To fully establish which stage of numerical magnitude representation and processing literacy affects, further studies are necessary-for example, number priming paradigms, which are thought to reveal features of numerical magnitude representation, rather than operations on these representations (such as comparison).

A final recommendation for future studies that examine the cultural influences on numerical cognition among illiterates is to develop a more detailed understanding of illiterate numeracy skills, either through the administration of normed numeracy assessments specifically designed for illiterate participants or, alternatively, through ethnographic observation. Given that our findings suggest that literates process symbolic magnitudes differently than do minimally literate individuals, an important next step is to examine which specific learning experiences bring about this change.

Taken together, the present findings strengthen the notion that the processing of nonsymbolic numerical magnitude taps into a basic system of magnitude representation that exhibits both phylogenetic and ontogenetic continuity. In addition to providing convergent evidence concerning the cultural invariance of nonsymbolic number processing, the present data extend our understanding of the effects of culture on symbolic and nonsymbolic magnitude processing by providing the first clear demonstration of the effect of literacy, independently of chronological age, on the processing of symbolic representations of numerical magnitude.

Acknowledgments This research was supported by a Discovery Grant from the National Sciences and Engineering Council of Canada and the Canada Research Chairs Program to DA.

\section{References}

Ansari, D. (2008). Effects of development and enculturation on number representation in the brain. Nature Reviews Neuroscience, 9, 278291.

Ardila, A., Berolucci, P., Braga, L., Castro-Caldas, A., Judd, T., Kosmidis, M., et al. (2010). The neuropsychology of cognition without reading. Archives of Clinical Neuropsychology, 25, 689-712.

Butterworth, B., Reeve, R., Reynolds, F., \& Lloyd, D. (2008). Numerical thought with and without words: Evidence from indigenous Australian children. Proceedings of the National Academy of Sciences, 105, 13179-13184.

Gordon, P. (2004). Numerical cognition without words: Evidence from Amazonia. Science, 206, 496-499.

Holloway, I. D., \& Ansari, D. (2009). Mapping numerical magnitudes onto symbols: The numerical distance effect and individual differences in children's mathematics achievement. Journal of Experimental Child Psychology, 103, 17-29.

Li, G., Cheung, R. T., Gao, J. H., Lee, T. M., Tan, L. H., Fox, P. T., et al. (2006). Cognitive processing in Chinese literate and illiterate subjects: An fMRI study. Human Brain Mapping, 27, $144-152$.

Nieder, A., \& Dehaene, S. (2009). Representation of number in the brain. Annual Review of Neuroscience, 32, 185-208.

Petersson, K. M., Reis, A., \& Ingvar, M. (2001). Cognitive processing in literate and illiterate subjects: A review of some recent behavioral and functional neuroimaging data. Scandinavian Journal of Psychology, 42, 251-267.

Pica, P., Lemer, C., Izard, V., \& Dehaene, S. (2004). Exact and approximate arithmetic in an Amazonian indigene group. Science, 306, 499-503.

Rouder, J. N., Speckman, P. L., Sun, D., Morey, R. D., \& Iverson, G. (2009). Bayesian $t$ tests for accepting and rejecting the null hypothesis. Psychonomic Bulletin \& Review, 16, 225-237.

Sekuler, R., \& Mierkiewicz, D. (1977). Children's judgments of numerical inequality. Child Development, 48, 630-633.

Van Opstal, F., Gevers, W., De Moor, W., \& Verguts, T. (2008). Dissecting the symbolic distance effect: Comparison and priming effects in numerical and nonnumerical orders. Psychonomic Bulletin \& Review, 15, 419-425.

Verguts, T., \& Fias, W. (2004). Representation of number in animals and humans: A neural model. Journal of Cognitive Neuroscience, 16, 1493-1504. 